\section{Wishful thinking: antidepressant drugs in childhood}

\section{depression}

ANNE TONKIN and JON JUREIDINI

The use of selective serotonin reuptake inhibitors (SSRIs) in children under 18 years old increased ten-fold (from 0.5 to 4.6 per 1000) in the UK from 1992 to 2001 (Murray et al, 2004). Usage rates are even higher in the USA, at 16.6 per 1000 (Delate et al, 2004), despite the existence of training and theoretical models for the treatment of mental disorders that are similar to those in the UK. Reasons for the increasing rates of use are likely to include heavy promotion of both medication and illness, distortion of the published data related to safety and efficacy, and underestimation by clinicians of the importance of the placebo response (Jureidini et al, 2004a). Over the past 2 years, serious concerns have been raised about the benefit-to-harm ratio of all antidepressants for children, leading to limits on the use of some drugs and warning statements on drug labelling. Nevertheless, a view persists that scepticism about these drugs is misplaced. Unfortunately this view, which owes more to wishful thinking than science, has permeated guidelines produced by professional and regulatory authorities, particularly in the USA. On what basis are these recommendations being made?

The evidence for efficacy has been disappointing. At least five unpublished trials using a placebo control have failed to show an advantage for antidepressants over placebo. Among eight published trials, four found no statistically significant advantage for antidepressants over placebo on any primary outcome measure, and only about a third (17/52) of all published measures show an advantage for drug over placebo. Even the statistically significant improvements are of dubious clinical importance; for example, Wagner et al (2003) showed that the Children's Depression Rating Scale - Revised (CDRS-R) score was reduced by 22.8 points by the use of sertraline compared with 20.2 points with placebo $(P=0.007)$. Thus we can have a high degree of confidence that there is an extremely small, and probably clinically unimportant, benefit from sertraline. It is also important to note that greater significance applied to adolescents rather than younger age groups, a point that was not included in the abstract of the article (Wagner et al, 2003). In discussing their own data, the authors of all of the four positive studies exaggerated the benefits, downplayed the harms, or both (Jureidini et al, 2004a). The pharmaceutical industry has further distorted the literature through suppression of data and ghost-writing of manuscripts. The most recently published study, the Treatment for Adolescents with Depression Study, like others before it, makes claims that are not supported by the data published in the paper (Jureidini et al, 2004b; Treatment for Adolescents with Depression Study Team, 2004). In an unmasked comparison that failed to account for placebo effects, expectancy effects and other non-pharmacological factors, cognitive-behavioural therapy with concurrent use of fluoxetine was more effective than cognitive-behavioural therapy alone. The most telling finding from this study was the lack of a statistical advantage of fluoxetine over placebo in the masked comparison on a primary endfinding was not mentioned in the abstract and has not been widely publicised.

In the absence of safety concerns even small improvements might make treatment worthwhile, but these drugs may occasionally cause serious adverse outcomes. In response to concerns raised about suicidal behaviour and withdrawal effects, the UK Committee on Safety of Medicines (CSM) established an expert panel in 2002 to review paroxetine and other SSRIs. Access to all GlaxoSmithKline's trials of paroxetine therapy revealed that the unpublished trials in children showed increased suicide risk and little evidence of efficacy. In fact, a GlaxoSmithKline internal memorandum point, the CDRS-R score $(P=0.10)$. This showed that the company had known that their studies failed to demonstrate efficacy since at least 1998. After reconstitution because of potential conflicts of interest, the expert panel reported in December 2003, and the CSM declared all SSRIs, except fluoxetine, contraindicated for use in patients under 18 years old.

The US Food and Drug Administration (FDA) was slower to take action. In January 2003, the FDA had approved fluoxetine as the first such drug for use in paediatric depression, despite only weak data for efficacy. By October 2003, the FDA had issued a 'Public Health Advisory' alerting physicians to reports of suicidal thinking and suicide attempts. A regulatory authority usually requires proof of efficacy, but in the face of an acknowledged lack of evidence, the FDA reversed the usual burden of proof, stating: 'failure to show effectiveness in any particular study... is not definitive evidence that the drug is not effective' (Food and Drug Administration, 2003). As Garland (2004) points out, a clinically significant response should be evident in a small trial, but detecting a low-frequency event such as suicidal behaviour is difficult in clinical trials. Yet two independent, FDA-commissioned reanalyses of the trial data showed that antidepressants almost doubled the risk of suicide-related events compared with placebo (risk ratio 1.81, 95\% CI 1.242.64). In October 2004, the FDA instructed manufacturers to include 'black box' warnings on all antidepressants about the risks of suicidal thoughts and behaviours.

Given that SSRIs have been marketed for many years, one might have expected a warning signal from post-marketing surveillance and reporting systems. Unfortunately post-marketing surveillance is poorly implemented. The 'yellow card' system in the UK is subject to underreporting, miscoding and flawed analyses (Medawar \& Herxheimer, 2003/2004), and FDA surveillance has been weakened by a shift of emphasis since drug companies began paying a premium to it in return for faster drug approvals, as the events surrounding the recent withdrawal of rofecoxib demonstrate. In the case of depression and suicidality, the waters are muddied by the overlap in symptoms between the condition and the drug, each of which may lead to suicidal ideation and behaviour, but it is safe to assume that there has been underestimation of the risk 
of suicidal behaviour associated with antidepressant drugs.

So, in the light of the evidence for a statistically significant but clinically insignificant benefit, and a low-frequency but very serious potential for harm, why have academic bodies continued to endorse the use of SSRIs in children? For example, as recently as October 2004, the American Association of Child and Adolescent Psychiatrists, on its website, urged their members 'to continue treating depressed children and adolescents with SSRI antidepressants' (American Association of Child and Adolescent Psychiatrists, 2004). The answer is probably related to how guidelines are developed and by whom. Often the clinicians involved are those with the most experience in using particular treatments, who have frequently been involved in industry-supported clinical trials and who have experienced success using these drugs without always recognising that more than $80 \%$ of this apparent benefit is probably related to the placebo effect. In many instances, the potential conflicts of interest inherent in this situation have not been addressed adequately.

Senior child psychiatrists warn us of the 'risk of doing nothing' (Brent, 2004), despite the evidence that psychotherapy has proved to be effective for childhood depression, and work by Gledhill et al (2003) suggests that there are feasible approaches for general practitioners to use in milder cases that involve neither drugs nor formal cognitive-behavioural therapy. Further, few children prescribed antidepressants persist with treatment for more than 2 months (Shireman et al, 2002; Murray et al, 2004), suggesting that the therapeutic benefits may not be sufficient to outweigh disadvantages in the view of the child or parent. It seems that, given the perceived need to 'do something' and the wishful thinking that the drugs may actually be better than the trial evidence indicates, the injunction to 'first do no harm' has been forgotten.

ANNE TONKIN, Department of Clinical and Experimental Pharmacology, University of Adelaide, JON JUREIDINI, Department of Psychological Medicine, Women's and Children's Hospital, North Adelaide, Australia

Correspondence: Dr AnneTonkin, Department of Clinical and Experimental Pharmacology, University of Adelaide, SA 5005, Australia.E-mail: anne.tonkin@adelaide.edu.au

(First received 17 December 2004, final revision 13 March 2005, accepted 30 March 2005)

The currently available evidence indicates that the SSRIs should not be recommended as first-line treatment in children with depression. We are concerned that regulators and writers of clinical guidelines worldwide will not grasp this nettle, particularly given the potential conflicts of interest of those who are also investigators in clinical trials. We are encouraged by the draft guidelines issued on its website on 23 November 2004 by the National Institute for Clinical Excellence (National Collaborating Centre for Mental Health, 2004), and hope that its final recommendations will not be marred by wishful thinking.

\section{DECLARATION OF INTEREST}

None. J.J. is Chairman of Healthy Skepticism, an organisation whose goal is to promote the appropriate use of drugs.

\section{REFERENCES}

American Association of Child and Adolescent Psychiatrists (2004) News Advisory on Antidepressant Message to AACAP Members II/5/04. http: / /www. aacap.org/Announcements/pdfs/SSRlemaill0_29_04I.pdf

Brent, D. A. (2004) Antidepressants and pediatric depression - the risk of doing nothing. New England Journal of Medicine, 35I, 1598-160।.

Delate, T., Gelenberg, A. J., Simmons, V. A., et al (2004) Trends in the use of antidepressants in a national sample of commercially insured pediatric patients, 1998 to 2002. Psychiatric Services, 55, 387-391.

Food and Drug Administration (2003) Reports of Suicidality in Pediatric Patients Being Treated with Antidepressant Medication for Major Depressive Disorder (MDD).Washington, DC: Center for Drug Evaluation and Research. http://www.fda.gov/cder/drug/ advisory/mdd. htm
Garland, E. J. (2004) Facing the evidence: antidepressant treatment in children and adolescents. Canadian Medical Association Journal, 170, 489-491.

Gledhill, J., Kramer, T., lliffe, S., et al (2003) Brief report: training general practitioners (GPs) in the identification and management of adolescent depression within the consultation: a feasibility study. Journal of Adolescence, 26, 245-250.

Jureidini, J., Doecke, C., Mansfield, P., et al (2004a) Efficacy and safety of antidepressants for children and adolescents. BMJ, 328, 879-883.

Jureidini, J., Tonkin, A. \& Mansfield, P. (2004b) TADS study raises concerns. BMJ, 329, 1343 .

Medawar, C. \& Herxheimer, A. (2003/2004) A comparison of adverse drug reaction reports from professionals and users, relating to risk of dependence and suicidal behaviour with paroxetine. International Journal of Risk and Safety in Medicine, 16, 5-19.

Murray, M. L., de Vries, C. S. \& Wong, I. C. (2004) A drug utilisation study of antidepressants in children and adolescents using the General Practice Research Database. Archives of Disease in Childhood, 89 , 1098-1102.

National Collaborating Centre for Mental Health (2004) Depression in Children: Identification and Management of Depression in Children and Young People in Primary Care and Specialist Services. http: //www. nice.org.uk/pdf/Depn_child_Istcons_Fullguideline.pdf

Shireman, T. I., Olson, B. M. \& Dewan, N. A. (2002) Patterns of antidepressant use among children and adolescents. Psychiatric Services, 53, 1444-1450.

Treatment for Adolescents with Depression Study Team (2004) Fluoxetine, cognitive-behavioral therapy, and their combination for adolescents with depression: Treatment for Adolescents with Depression Study (TADS) Randomized Controlled Trial. JAMA, 292 807-820.

Wagner, K. D., Ambrosini, P., Rynn, M., et al (2003) Efficacy of sertraline in the treatment of children and adolescents with major depressive disorder: two randomized controlled trials. JAMA, 290, 1033-104I.

Whittington, C. J., Kendall, T., Fonagy, P., et al (2004) Selective serotonin reuptake inhibitors in childhood depression: systematic review of published versus unpublished data. Lancet, 363, I34I-1345. 